\title{
Modulated segregation of a bidisperse granular mixture due to recirculation currents
}

\author{
Kwami Adem Mayeden ${ }^{1 *}$,Evelyne Kolb ${ }^{2}$, Stéphanie Deboeuf ${ }^{3}$, Pierre Jop ${ }^{1}$ \\ ${ }^{1}$ SVI (Surface du Verre et Interfaces), CNRS/SAINT-GOBAIN RESEARCH PARIS, 93300 Aubervilliers, France \\ ${ }^{2}$ PMMH (Physique et Mécanique des Milieux Hétérogènes), UMR 7636, 75005, Paris, France \\ ${ }^{3}$ Institut Jean Le Rond d'Alembert, Place Jussieu 75252 Paris Cedex 05, Paris, France
}

\begin{abstract}
A bidisperse medium of large sand particles and small glass beads is mixed in a cylindrical vessel that is put in rotational motion around an eccentric static rod. The subsequent segregation occurring in the system is investigated primarily at the free-surface where the large particles concentration globally increases towards a limit value while oscillating. In order to gain deeper understanding of the intrinsic mechanisms causing the segregation, we also explore the dynamics in the bulk. Average residual bulk displacements fields both vertically and orthoradially could then be measured and show a secondary flow that counteracts the segregation process.
\end{abstract}

\section{Introduction}

Ubiquitous in a wide range of applications, granular materials have been receiving over the past decades a growing attention in the research community in order to establish their rheology $[1,2]$. One of the main focuses, especially in the engineering world, is the problem of particles mixing. It raises interesting and fundamental problematics on interaction between particles of different properties; one of the recurring being segregation, mainly size-segregation [3, 4] that often leads to structural mal-performance in industrial products such as glass, abrasives, pills just to name a few.

Even though the dedication of the granular community has allowed to establish theoretical [5] and phenomenological [6] laws describing the phenomenon, with recent numerical insights [7], it has to be noticed that these findings remain solely applicable to classical configurations such as the rotating drum, the heap flow, the flow down an inclined plane or the Couette-flow. The generalization to complex flows remains an open question. In this paper we experimentally investigate segregation in one of these complex flows generated by the rotation of a cylindrical vessel around a fixed eccentric rod. We start off by focusing on segregation dynamics at the free-surface before investigating the flow behavior in the bulk.

\section{Experimental Setup}

The setup (Fig. 1) consists in a cylindrical vessel of diameter $D=14 \mathrm{~cm}$ in which a bidisperse medium of particles is mixed: large sand particles of average diameter $d_{l}=600 \mu \mathrm{m}$ colored in green and white small glass beads ( $d_{s}=250 \mu \mathrm{m}$ ). For the mixing, we use a rod in steel of diameter $d_{\text {rod }}=2 \mathrm{~cm}$ whose center is positioned at $2 \mathrm{~cm}$ from the wall - the immersion depth of the rod can be varied but for the experiments mentioned below, its end-base was kept at $2 \mathrm{~cm}$ from the bottom of the vessel. To start the mixing, the vessel is put in rotational motion at $\omega \approx 1.5 \mathrm{rpm}$ while the rod is kept fixed. Such an angular velocity allows to stay in a quasi-static flow regime with an inertial number around $4.10^{-4}$ in which the shear rate is estimated from the rod diameter, the rod velocity and the average depth pressure.
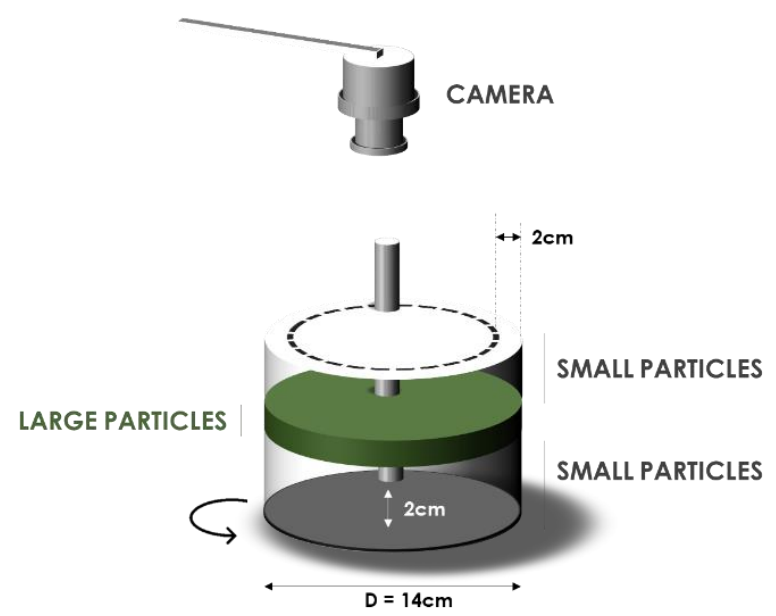

Fig. 1 : Experimental setup

The purpose of the experiment being to investigate particle size segregation in the medium, we started with a stratified arrangement with, from the bottom to the top of the vessel: a $3 \mathrm{~cm}$ thick layer of small particles then 
a $2-4 \mathrm{~mm}$ thick layer of large particles and to end the stack another $2 \mathrm{~cm}$ thick layer of small particles. The bottom layer of small particles was thick enough to avoid boundary-effects from the bottom of the vessel. Once the mixing starts, we use a camera affixed at the top of the vessel to capture 8 bit grayscale images (2048x2048 pixels) of the free-surface at an acquisition rate of $0.5 \mathrm{~Hz}$. We surround the setup with a ring of red LEDs to improve the contrast between large green particles and small white particles at the surface. On a typical image, one could see hence large particles in black and small particles in white. As mixing goes on, segregation occurs and large particles are seen to rise progressively to the free-surface (Fig. 2) until a stationary pattern occurs.

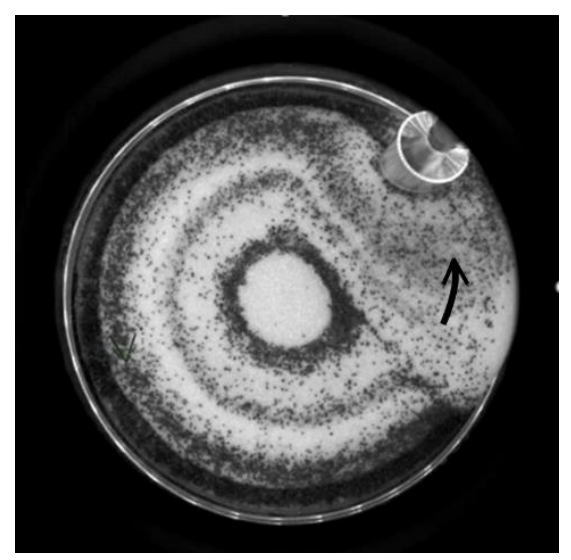

Fig. 2: Typical picture of the free-surface after 15 turns with large particles (appearing black) that have segregated, and small particles appearing in white. Initially the top surface was only covered with small white particles.

\section{Analysis of the surface to quantify the segregation}

We investigate segregation by processing the successive images that were captured by the camera through time. When rotation occurs, dark large particles rise to the free-surface and the corresponding average grayscale intensity value of the free surface decreases. In order to characterize this process, we define a coverage-ratio $C(t)$ as follows :

$$
C(t)=\frac{I_{0}-I(t)}{I_{0}-I_{f c}},
$$

where $I(t)$ is the average grayscale of the surface at time $\mathrm{t}(0=$ black and $255=$ white $), I_{0}$ the grayscale at the beginning of the experiment(when the whole free surface is covered with only small white particles) and $I_{f c}$ is the grayscale of the free-surface in a hypothetical scenario where the whole surface is covered with black large particles ( $f_{c}$ for full coverage). Note that $0<$ $I_{f c} \leq I(t) \leq I_{0}<255$. While experimentally, we never observe a full coverage with large particles, this definition of $C(t)$ allows to bound it between 0 (no large particles at surface) and 1 (full surface saturation with large particles) and to compare consistently different experiments.

\section{Results}

\subsection{Segregation: accumulation at the free- surface}

The plotting of $C(t)$ against time (Fig. 3) shows an overall increase with an exponential relaxation towards a stable regime modulated by repetitive oscillations of very long periods (roughly 16 turns). An exponential rise of large grains was already observed by L. Staron and J.C. Phillips [8] in a numerical two-dimensional bidisperse chute flow and by Golick and Daniels [3] in an experimental horizontal plane shear-flow. In both configurations, the application of a continuous solicitation leads to a particle segregation in the medium. In agreement with literature, such a segregation results in the full coverage with large particles at the surface of the flow $[3,8,9]$. In our configuration where the medium is also continuously sheared by the passing of the rod, one could expect a similar full coverage. However, even in the wake of the rod, where the shear rate is the highest, we observe only a partial coverage with large particles: large particles once at the surface do not necessarily stay atop, they can plunge back in the bulk and a pattern can be seen. The oscillations represent the successive disappearances and appearances of particles at the surface and are the signature of an entrainment of the particles from the surface towards the bulk of the system. This rise-andplunge motion due to a strong recirculation motion becomes less visible when an equilibrium state is reached in which the repartition of large particles at the surface and the exchanges between bulk and freesurface could be considered as stable. The dynamics observed in the medium through $C(t)$ can then be viewed as a competition between segregation and recirculation currents. In a recent work, D'Ortona and Thomas have shown a destabilisation of a segregated top layer of large grains due to their large density that produces also a pattern on chute flow and a partial coverage [10]. However, this mechanism is excluded in our experiment where both large and small particles have the same density.

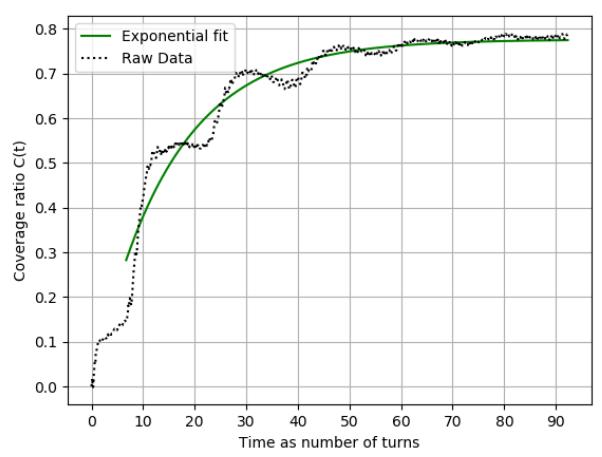

Fig. 3: Evolution of the coverage ratio $C(t)$ against time for the same parameters as in Fig. 1. 


\subsection{Segregation in the bulk against distance from the center of the rod}

In order to better understand the segregation dynamics, we design experiments to investigate its manifestation in the bulk.

A $2 \mathrm{~mm}$ layer of large particles is inserted between two layers of small particles, $3 \mathrm{~cm}$ at the bottom and $2 \mathrm{~cm}$ on top. Mixing is performed for 4 revolutions at an angular velocity of $\omega \approx 1.5 \mathrm{rpm}$. At the end, a vacuum cleaner is used to explore the bulk by removing particles layer by layer. Pictures of the inside could then be retrieved that show the repartition of particles radially given the depth. In Fig. 4, we observe such a pattern.

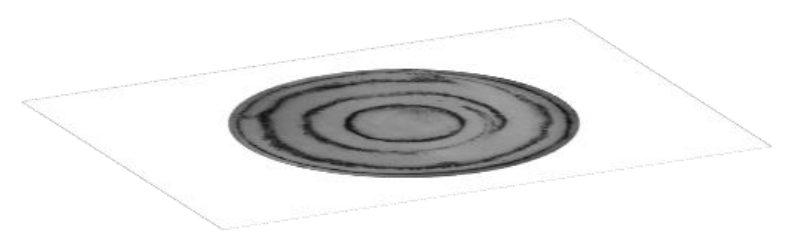

Fig. 4: Example of a slice taken at a height $z_{i}=4 \mathrm{~cm}$ after 4 revolutions in the experimental configuration shown in Fig. 1

For a given picture $i$ taken at a height $z_{i}$ from the bottom of the vessel (Fig. 4), the circular surface of the vessel is divided in concentric rings of radius $r$ and fixed width $\mathrm{d} r=3 \mathrm{~mm}$. The quantity of large particles on each ring is estimated by the following equation:

$$
M_{r, i}=\frac{I_{i}^{0}-I_{r, i}}{I_{i}^{0}-I_{f c}}
$$

where $I_{r, i}$ is the average grayscale of the ring of radius $r$ on a picture $i$ located at $z_{i}, I_{i}^{0}$ the average grayscale value of a reference area free from any large particle on the picture $i$, and $I_{f c}$ is still defined as in paragraph 3 . Using all the images ( $i$ between 0 an $n$ ) spaced vertically by $d z_{i}$, and the above-defined estimate, one could evaluate a barycenter of the vertical positions of the particles at $r$ after the 4 revolutions using ( 3 ).

$$
\overline{z_{r}}=\frac{\sum_{0}^{n} z_{i} * M_{r, i} \mathrm{~d} z_{i}}{\sum_{0}^{n} M_{r, i} \mathrm{~d} z_{i}}
$$

Plotting $\overline{z_{r}}$ against the normalized distance from the center of the rod gives hence an idea on the aptitude of particles to rise given their radial position (Fig. 5).

The minimal rise is obtained as expected far away from the rod in the central area of the vessel where the shear induced by the rod is less felt. In that area, the small non-zero value of the rise relatively to the initial position of the large particles deposit can be attributed to measurements error. The maximal rise on the other hand is obtained at a normalized distance of +1 , that is at a distance $\frac{d_{\text {rod }}}{2}$ from the outer surface of the rod towards the center of the vessel. If this rise was only due to the amplitude of the shear as usually modeled in literature [4], one would expect the maximal rise to occur close to the rod's surface (at a normalized distance around 0.5 where the shear is expected to be maximal). This result supports the fact that the segregation process is not the only mechanism responsible for the vertical movement of the grains.

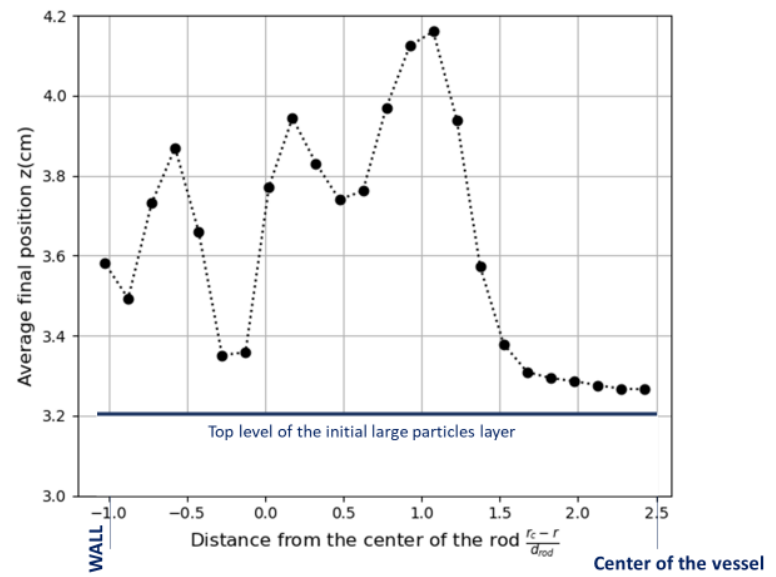

Fig. 5: Average vertical displacement field against normalized distance from the center of the rod after 4 revolutions (the top level of the layer of large particles was initially located at $3.2 \mathrm{~cm}$ from the bottom of the vessel) $-r_{c}$ being the radial coordinate of the center of the rod

The entire rise dynamics at the center part of the rod seems in nutshell to tend smoothly towards zero. On the wall side, a similar behavior can be observed only with a lower amplitude. On the path of the rod, the rise is not uniform. The point of minimal rise in that area does not coincide with the center of the rod and as one moves away from it, particles seems to segregate more. We attribute this again to the existence of a downward secondary flow localized behind the rod.

\subsection{Orthoradial displacement in the bulk against distance from the center of the rod}

As segregation is due to shear which itself stems from displacement, we set up an experiment to retrieve the orthoradial displacement generated by the passage of the rod in the depth of the medium (Fig. 6). We laid a thin bar of large particles in between two layers of small particles $(3 \mathrm{~cm}$ in the bottom and $2 \mathrm{~cm}$ above respectively). We rotate the vessel for one revolution and vacuumed the particles in order to retrieve the deformation of the bar (residual displacement) stemmed from the passing of the rod. We hence assumed only an orthoradial displacement (radial displacement negligible) and plot the curvilinear displacement $r *$ $\theta(\mathrm{cm})$ against the normalized distance from the center of the rod (Fig. 7).

We only focused on the displacement profile on the center-side part of the rod. Two main regions could be 
discriminated : the first, on the path of the rod and in its close vicinity and a second region far from the rod (above $0.7 d_{\text {rod }}$ ). Note that above $1.5 d_{\text {rod }}$ the curvilinear displacement is very low and it is experimentally difficult to measure it with precision. This explains the noise close to the center of the vessel. Measuring after more revolutions could improve this point.

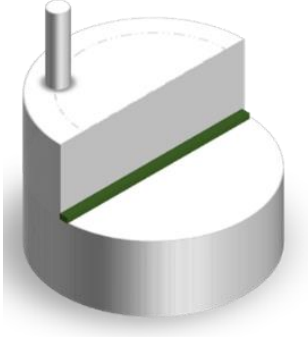

(a)

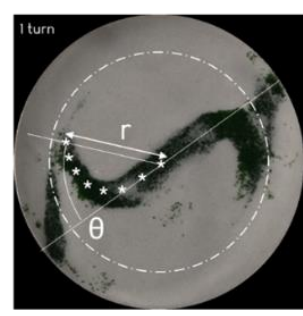

(b)
Fig. 6: (a) Illustration of the experimental protocol showing where the thin bar of large (green) particles is deposited (b) Deformed bar after one revolution

A linear fit matches the experimental data in the first region whereas an exponential decay satisfyingly fits the curvilinear displacement in the second region further away (inset Fig. 7) with a characteristic distance of $\delta=$ $0.22 d_{\text {rod }}$. The exponential displacement in the plane is consistent with previous measurements around an intruder $[11,12]$ or more generally when creeping flow occurs far from a perturbation (e.g. flows on a pile, wide Couette flows) [2]. The origin of the linear part still has to be unfolded: we observe indeed only the final position and the complete path of the grains during the passing of the rod should be measured. We expect the crossover between those regimes to correspond to the rod diameter.

The shear stemming from this orthoradial displacement profile should be related to the segregation and thus to the rise of the grains: this is the case far away from the rod where the one decade rise variation between $1.2 d_{\text {rod }}$ and $1.7 d_{\text {rod }}$ (Fig. 5) has the same characteristic distance $\delta$. Above $1.7 d_{\text {rod }}$, very close to the center of the vessel, the quasi-null rise makes it difficult to establish a precise comparison. And below $1.2 d_{\text {rod }}$ going into the vicinity of the rod, since the orthoradial displacement curve is monotonous contrary to the rise curve, this tends to confirm again the existence of another mechanism in the medium that intervenes in the vertical motion of the particles: the recirculation current [13-15] whose characterization in our configuration is being carried on. So far, we suspect it to be due to either the falling down of particles at the free surface in the wake behind the rod or the variation of the local volume fraction [15].

\section{Conclusion}

We investigated segregation dynamics occurring in a bidisperse medium mixed in a cylindrical vessel with an eccentric rod. We showed that the relaxation law towards a steady state has an exponential baseline that is modulated by periodic oscillations which point to the existence of recirculation currents. In the bulk, we studied the rise of large particles according to their radial positions. Last, we explored the orthoradial displacement field inside the medium generated by the passing of the rod and showed that it follows an exponential law that is correlated with the rise of large particles far away from the rod. More investigation has to be done especially to understand the downward movement behind the rod and the coupling of recirculation currents with the segregation mechanism.

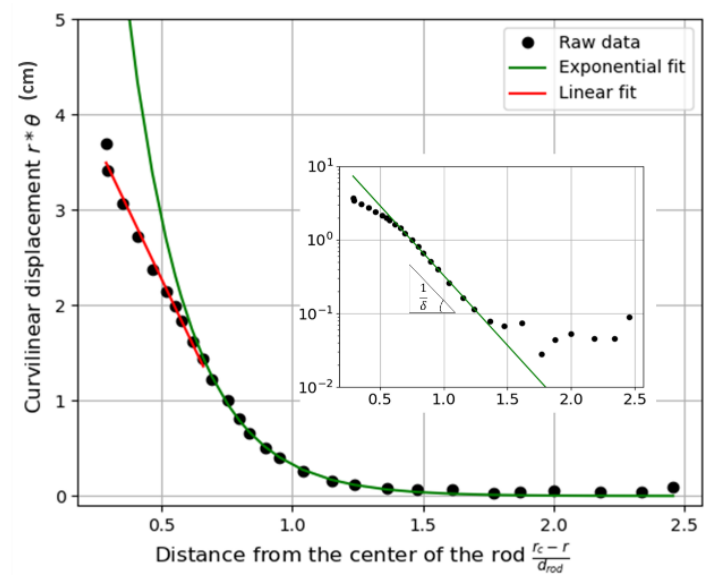

Fig. 7: Curvilinear displacement against distance from the center of the rod - graph in log space in the inset

\section{References}

[1] P. Jop, Y. Forterre, O. Pouliquen, Nature 441, 727-30 (2006)

[2] MiDi, G.D.R., Eur. Phys. J. E 14, 341-65 (2004)

[3] L.A. Golick, K.E. Daniels, Phys. Rev. E 80, 042301 (2009)

[4] J.M.N.T. Gray, Annu. Rev. Fluid Mech. 50, 407-433 (2018)

[5] J.M.N.T. Gray, C. Ancey, J. Fluid. Mech. 678, 535-588 (2011)

[6] P. Gajjar, J.M.N.T. Gray, J. Fluid. Mech. 757, 297-329 (2014)

[7] F. Guillard, Y. Forterre, O. Pouliquen, J. Fluid. Mech. 807, R1 (2016)

[8] L. Staron, J.C. Phillips, Phys. Fluids 26, 033302 (2014)

[9] K. van der Vaart, et al., Phys. Rev. Lett. 114, 238001 (2015)

[10] U. D'Ortona, N. Thomas, Phys. Rev. Lett. 124, 178001 (2020)

[11] A. Seguin, et al., Phys. Rev. E 93, 012904 (2016)

[12] E. Kolb, et al., Phys. Rev. E 87, 032207 (2013)

[13] E.E. Ehrichs, et al., Science 267, 1632-1634 (1995)

[14] S.S. Hsiau, P.C. Wang, C.H. Tai, AIChE Journal 48, 1430-1438 (2002)

[15] K.P. Krishnaraj, P.R. Nott, Nat. Commun. 7, 10630 (2016) 